\title{
SOME GENERALIZATIONS OF GREGORY'S POWER SERIES AND THEIR APPLICATIONS
}

\author{
Natalia Gawrońska, Damian Stota, Roman Wituła, Adam Zielonka \\ Institute of Mathematics, Silesian University of Technology \\ Gliwice, Poland \\ damian.slota@polsl.pl
}

\begin{abstract}
The series representing the generalizations of classical James Gregory's series are discussed in this paper. Formulae describing sums of these series are found. A number of applications of obtained formulae are also presented, among others, in receiving the generalizations of Gregory-Leibniz-Nilakantha formula. Moreover, the sums of series of differences of odd harmonic numbers are generated.
\end{abstract}

Keywords: Gregory's series, Gregory-Leibniz-Nilakantha formula

AMS Subject Classification: 40A30, 40A05, 40-04.

\section{Introduction}

James Gregory (1638-1675), Scottish mathematician, discovered the Taylor series more than forty years before Taylor published it. Also the Maclaurin series for $\tan x, \sec x, \arctan x$ and sec $x$ were all known to him, but only the series for $\arctan x(1671)$ :

$$
\arctan x=x-\frac{x^{3}}{3}+\frac{x^{5}}{5}-\frac{x^{7}}{7}+\cdots, \quad|x| \leq 1,
$$

is known as "Gregory's series" [1]. This series, by applying it into formula

$$
\frac{\pi}{4}=4 \arctan \frac{1}{5}-\arctan \frac{1}{239}
$$

discovered in 1706 by English mathematician John Machin (1680-1752), made it possible to obtain the relation

$$
\begin{aligned}
\pi= & \frac{16}{5}\left(1-\frac{4}{3 \cdot 100}+\frac{4^{2}}{5 \cdot 100^{2}}-\frac{4^{3}}{7 \cdot 100^{3}}+\cdots\right)- \\
& -\frac{4}{239}\left(1-\frac{1}{3 \cdot 57121}+\frac{1}{5 \cdot 57121}-\cdots\right)
\end{aligned}
$$


used by Machin for numerical calculating the value of $\pi$ (exact to one hundred decimal places) which broke the hegemony of the geometrical method of Archimedes used from ancient times for determining the approximations of number $\pi$.

The aim of our paper is to give some generalizations of formula (1). We distinguish an infinite family of such generalizations and we present their various applications, among others, in generating new formulae of numerical nature. In particular, we give the generalizations of the classic Gregory-Leibniz-Nilakantha formula [2]:

$$
\frac{\pi}{4}=1-\frac{1}{3}+\frac{1}{5}-\frac{1}{7}+\cdots
$$

and their applications for summation of series of differences of odd harmonic numbers. Formula (2) was historically the first formula in which $\pi$ represented the sum of numerical series.

The subject matter concerning the summation of the series was already an object of the authors' interests, the effect of which is given in paper [3]. In this paper the Fourier series method, elementary trigonometric and the residue method were used. For a change, in the current paper we use only the simple calculus method and an elementary trigonometric one. It appears that in paper [3], as well as in the presented paper, apart from new results, relations and identities, there are discovered a lot of inexhaustible, still inspiring subjects which will certainly lead to prepare new works.

\section{Generalizations of Gregory's formula}

We begin with determining the generalizations of Gregory alternating power series (right hand of (1)). Let us take

$$
\begin{gathered}
S_{1}(x)=x-\frac{x^{3}}{3}+\frac{x^{5}}{5}-\frac{x^{7}}{7}+\cdots \\
S_{2}(x)=x+\frac{x^{3}}{3}-\frac{x^{5}}{5}-\frac{x^{7}}{7}+\frac{x^{9}}{9}+\frac{x^{11}}{11}-\frac{x^{13}}{13}-\frac{x^{15}}{15}+\cdots
\end{gathered}
$$

and, in general

$$
\begin{gathered}
S_{n}(x)=x+\frac{x^{3}}{3}+\cdots+\frac{x^{2 n-1}}{2 n-1}-\frac{x^{2 n+1}}{2 n+1}-\frac{x^{2 n+3}}{2 n+3}-\cdots-\frac{x^{4 n-1}}{4 n-1}+ \\
+\frac{x^{4 n+1}}{4 n+1}+\frac{x^{4 n+3}}{4 n+3}+\cdots+\frac{x^{6 n-1}}{6 n-1}-\frac{x^{6 n+1}}{6 n+1}-\cdots-\frac{x^{8 n-1}}{8 n-1}+\cdots
\end{gathered}
$$

By using the possibility of integrating the power series (by treating every term separately) in the interval of convergence, we easily get the following relation for each $x \in[-1,1]$ : 


$$
\begin{gathered}
S_{n}(x)=\int_{0}^{x}\left(1+t^{2}+\cdots+t^{2 n-2}-t^{2 n}-t^{2 n+2}-\cdots-t^{4 n-2}+t^{4 n}+t^{4 n+2}+\cdots\right. \\
=\int_{0}^{x}\left(1+t^{2}+\cdots+t^{2 n-2}\right)\left(1-t^{2 n}+t^{4 n}-t^{6 n}+\cdots\right) d t= \\
=\int_{0}^{x} \frac{1+t^{2}+\cdots+t^{2 n-2}}{1+t^{2 n}} d t=\int_{0}^{x} \frac{1-t^{2 n}}{\left(1-t^{2}\right)\left(1+t^{2 n}\right)} d t=
\end{gathered}
$$

for $n=1$ :

$$
=\arctan x \text {, }
$$

for $n=2$ :

$$
=\frac{\sqrt{2}}{2}(\arctan (\sqrt{2} x-1)+\arctan (\sqrt{2} x+1))
$$

for $n=3$ :

$$
=\frac{1}{3} \arctan x-\frac{2}{3} \arctan \left(\frac{x}{x^{2}-1}\right) \text {, }
$$

for $n=4$ :

$$
\begin{aligned}
= & \frac{\sqrt{2}}{2}\left(\cos \frac{\pi}{8}\left(\arctan \left(x \csc \frac{\pi}{8}-\cot \frac{\pi}{8}\right)+\arctan \left(x \csc \frac{\pi}{8}+\cot \frac{\pi}{8}\right)\right)+\right. \\
& \left.+\sin \frac{\pi}{8}\left(\arctan \left(x \sec \frac{\pi}{8}-\tan \frac{\pi}{8}\right)+\arctan \left(x \sec \frac{\pi}{8}+\tan \frac{\pi}{8}\right)\right)\right),
\end{aligned}
$$

for $n=5$ :

$$
\begin{gathered}
=\frac{1}{5}\left(\arctan x+4 \cos \frac{2 \pi}{5}\left(\arctan \left(x \operatorname{scs} \frac{\pi}{5}-\tan \frac{\pi}{5}\right)+\arctan \left(x \operatorname{scs} \frac{\pi}{5}+\tan \frac{\pi}{5}\right)\right)+\right. \\
\left.+4 \cos \frac{\pi}{5}\left(\arctan \left(x \operatorname{scs} \frac{2 \pi}{5}+\tan \frac{2 \pi}{5}\right)+\arctan \left(x \operatorname{scs} \frac{2 \pi}{5}-\tan \frac{2 \pi}{5}\right)\right)\right)= \\
=\frac{1}{5}\left(\arctan x+(\sqrt{5}-1)\left(\arctan \left(\frac{4 x-\sqrt{10-2 \sqrt{5}}}{\sqrt{5}+1}\right)+\right.\right. \\
\left.+\arctan \left(\frac{4 x+\sqrt{10-2 \sqrt{5}}}{\sqrt{5}+1}\right)\right)+ \\
\left.+(\sqrt{5}+1)\left(\arctan \left(\frac{4 x+\sqrt{10+2 \sqrt{5}}}{\sqrt{5}-1}\right)+\arctan \left(\frac{4 x-\sqrt{10+2 \sqrt{5}}}{\sqrt{5}-1}\right)\right)\right)
\end{gathered}
$$


for $n=6$ :

$$
\begin{gathered}
=\frac{\sqrt{2}}{6}(\arctan (\sqrt{2} x+1)+\arctan (\sqrt{2} x-1)+ \\
+(\sqrt{3}+1)\left(\arctan \left(\frac{2 \sqrt{2} x-1-\sqrt{3}}{\sqrt{3}-1}\right)+\arctan \left(\frac{2 \sqrt{2} x+1+\sqrt{3}}{\sqrt{3}-1}\right)\right)+ \\
\left.+(\sqrt{3}-1)\left(\arctan \left(\frac{2 \sqrt{2} x+\sqrt{3}-1}{\sqrt{3}+1}\right)+\arctan \left(\frac{2 \sqrt{2} x-\sqrt{3}+1}{\sqrt{3}+1}\right)\right)\right)= \\
=\frac{1}{3} S_{2}(x)+\frac{\sqrt{2}}{6}\left(( \sqrt { 3 } + 1 ) \left(\arctan \left(\frac{2 \sqrt{2} x-1-\sqrt{3}}{\sqrt{3}-1}\right)+\right.\right. \\
+(\sqrt{3}-1)\left(\arctan \left(\frac{2 \sqrt{2} x+1+\sqrt{3}}{\sqrt{3}-1}\right)\right)+ \\
\left.\left.+\left(\frac{2 \sqrt{2} x+\sqrt{3}-1}{\sqrt{3}+1}\right)+\arctan \left(\frac{2 \sqrt{2} x-\sqrt{3}+1}{\sqrt{3}+1}\right)\right)\right)
\end{gathered}
$$

for $n=7$ :

$$
\begin{gathered}
=\frac{1}{7}\left(\arctan x+\left(1+2 \cos \frac{\pi}{7}-2 \sin \frac{\pi}{14}-2 \sin \frac{3 \pi}{14}\right) \times\right. \\
\times\left(\arctan \left(x \sec \frac{\pi}{7}-\tan \frac{\pi}{7}\right)+\arctan \left(x \sec \frac{\pi}{7}+\tan \frac{\pi}{7}\right)\right)+ \\
+\left(-1+2 \cos \frac{\pi}{7}-2 \sin \frac{\pi}{14}+2 \sin \frac{3 \pi}{14}\right)\left(\arctan \left(x \csc \frac{3 \pi}{14}-\cot \frac{3 \pi}{14}\right)+\right. \\
\left.+\arctan \left(x \csc \frac{3 \pi}{14}+\cot \frac{3 \pi}{14}\right)\right)+ \\
+\left(1+2 \cos \frac{\pi}{7}+2 \sin \frac{\pi}{14}+2 \sin \frac{3 \pi}{14}\right) \times \\
\left.\times\left(\arctan \left(x \csc \frac{\pi}{14}-\cot \frac{\pi}{14}\right)+\arctan \left(x \csc \frac{\pi}{14}+\cot \frac{\pi}{14}\right)\right)\right),
\end{gathered}
$$

for $n=9$ : 


$$
\begin{aligned}
& =\frac{2}{9}\left(\frac{1}{2} \arctan x+\arctan (2 x+\sqrt{3})+\arctan (2 x-\sqrt{3})+\right. \\
& +\left(-1+\cos \frac{\pi}{9}+\cos \frac{2 \pi}{9}-\sin \frac{\pi}{18}\right) \times \\
& \times\left(\arctan \left(x \sec \frac{\pi}{9}-\tan \frac{\pi}{9}\right)+\arctan \left(x \sec \frac{\pi}{9}+\tan \frac{\pi}{9}\right)\right)+ \\
& +\left(1-\cos \frac{\pi}{9}+\cos \frac{2 \pi}{9}-\sin \frac{\pi}{18}\right) \times \\
& \times\left(\arctan \left(x \sec \frac{2 \pi}{9}-\tan \frac{2 \pi}{9}\right)+\arctan \left(x \sec \frac{2 \pi}{9}+\tan \frac{2 \pi}{9}\right)\right)+ \\
& +\left(1+\cos \frac{\pi}{9}+\cos \frac{2 \pi}{9}+\sin \frac{\pi}{18}\right) \times \\
& \left.\times\left(\arctan \left(x \csc \frac{\pi}{18}-\cot \frac{\pi}{18}\right)+\arctan \left(x \csc \frac{\pi}{18}+\cot \frac{\pi}{18}\right)\right)\right) \text {. }
\end{aligned}
$$

One can see that formulae for $n=1,2,3,6$ are especially useful for computations. Formulae for $n=4,7,9$ are of a more advanced trigonometrical nature, however they can be used for calculations as well.

Remark 1. Let us notice that computing the values of $\arctan x$ for $x>1$ can be always reduced to computing the values of $\arctan y$ for $y \in(0,1)$, since the following identity [4]:

$$
\frac{1}{2} \arctan x=\arctan \frac{\sqrt{1+x^{2}}-1}{x}, \quad x>0
$$

is satisfied. Moreover, if we take

$$
f(x)=\frac{\sqrt{1+x^{2}}-1}{x}, \quad x>0
$$

then $f(x)<\frac{1}{2} x$ which implies that $\left(f^{n+1}:=f \circ f^{n}, n=1,2, \ldots\right)$ :

$$
\lim _{n \rightarrow \infty} f^{n}(x)=0
$$

for every $x>0$. Thus, for an appropriately quick computation of $\arctan x$ values, it is enough to apply Gregory's formula for $\arctan \left(f^{n}(x)\right)$ for sufficiently big numbers $n \in \mathbb{N}$, since

$$
\arctan x=2^{n} \arctan \left(f^{n}(x)\right), \quad x>0 .
$$


For example

$$
\arctan \frac{3}{4}=2 \arctan \frac{1}{3}=4 \arctan \frac{1}{\sqrt{10}+3}=\cdots
$$

Of course, it does not mean that the other formulae, given above, cannot be used. Quite the contrary, and, what is essential, the other formulae can give even better convergence.

\section{Review of applications of formulae (3)-(10)}

We present only the list of selected relations:

a)

$$
\begin{gathered}
S_{2}(1)=\frac{\sqrt{2}}{2}(\arctan (\sqrt{2}-1)+\arctan (\sqrt{2}+1))= \\
=\lim _{x \rightarrow 1^{-}} \frac{\sqrt{2}}{2}(\arctan (\sqrt{2} x-1)+\arctan (\sqrt{2} x+1))= \\
=\lim _{x \rightarrow 1^{-}} \frac{\sqrt{2}}{2}\left(\arctan \frac{2 \sqrt{2} x}{1-\left(2 x^{2}-1\right)}\right)= \\
=\lim _{x \rightarrow 1^{-}} \frac{\sqrt{2}}{2} \arctan \left(\frac{\sqrt{2} x}{1-x^{2}}\right)=\frac{\sqrt{2}}{2} \arctan (\infty)=\frac{\sqrt{2}}{4} \pi,
\end{gathered}
$$

that is

$$
\arctan (\sqrt{2}-1)+\arctan (\sqrt{2}+1)=\frac{\pi}{2}
$$

which is a special case of the known formula [2]:

$$
\arctan x+\arctan \frac{1}{x}=\frac{\pi}{2} \operatorname{sgn}(x), \quad x \in \mathbb{R} \backslash\{0\} .
$$

b)

$$
S_{2}\left(\frac{\sqrt{2}}{2}\right)=\frac{\sqrt{2}}{2} \arctan 2
$$

which implies the equality

$$
\arctan 2=\sum_{k=0}^{\infty}(-1)^{k} 4^{-k}\left(\frac{1}{4 k+1}+\frac{1}{2(4 k+3)}\right)
$$

From this and from Gregory's formula for $x=\frac{1}{3}$ and from the equality, which is easy to verify [4]: 


$$
2 \arctan 2-\arctan \frac{3}{4}=\frac{\pi}{2}
$$

i.e., by (11)

$$
\arctan 2-\arctan \frac{1}{3}=\frac{\pi}{4}
$$

we get two series, both of geometrical rate of convergence, linear combination of which approximates $\pi$.

c)

$$
S_{3}(1)=\frac{\pi}{12}-\frac{2}{3} \arctan (-\infty)=\frac{\pi}{12}-\frac{2}{3}\left(-\frac{\pi}{2}\right)=\frac{5}{12} \pi,
$$

which implies the equality

$$
\pi=\frac{12}{5} \sum_{k=1}^{\infty}(-1)^{k-1}\left(\frac{1}{6 k-5}+\frac{1}{6 k-3}+\frac{1}{6 k-1}\right) .
$$

Unfortunately, this alternating series is rather slowly convergent.

d)

$$
S_{3}\left(\frac{\sqrt{3}}{3}\right)=\frac{1}{3} \arctan \frac{\sqrt{3}}{3}+\frac{2}{3} \arctan \frac{\sqrt{3}}{2}=\frac{\pi}{18}+\frac{2}{3} \arctan \frac{\sqrt{3}}{2}
$$

which implies

$$
\frac{\pi}{6}+2 \arctan \frac{\sqrt{3}}{2}=3 \sqrt{3} \sum_{k=1}^{\infty}(-1)^{k-1} 27^{-k}\left(\frac{9}{6 k-5}+\frac{1}{2 k-1}+\frac{1}{6 k-1}\right) .
$$

We also have

$$
S_{3}\left(\frac{\sqrt{n}}{n}\right)=\frac{1}{3} \arctan \frac{\sqrt{n}}{n}+\frac{2}{3} \arctan \frac{\sqrt{n}}{n-1}
$$

which implies

$$
\begin{gathered}
\arctan \frac{\sqrt{n}}{n}+2 \arctan \frac{\sqrt{n}}{n-1}= \\
=3 \sqrt{n} \sum_{k=1}^{\infty}(-1)^{k-1} n^{-3 k}\left(\frac{n^{2}}{6 k-5}+\frac{n}{6 k-3}+\frac{1}{6 k-1}\right) .
\end{gathered}
$$


From this, by applying Gregory's series for $\arctan \frac{1}{k}, k>1$, we can also compute the value of $\arctan \frac{k}{k^{2}-1}$ in the convergence rate of geometrical series. Moreover, let us notice a curious detail - from equality [4]:

$$
\arctan \frac{1}{2}+\arctan \frac{1}{3}+\arctan \frac{2}{3}=\arctan 5,
$$

by applying formula (15) for $n=4$ and by using Gregory's formula twice for $x=\frac{1}{2}$ and $x=\frac{1}{3}$, we can calculate $\arctan 5$ in the convergence rate of geometrical series.

e)

$$
S_{3}\left(\frac{\sqrt{2}}{2}\right)=\frac{1}{3} \arctan \frac{\sqrt{2}}{2}+\frac{2}{3} \arctan \sqrt{2}
$$

which implies

$\arctan \frac{\sqrt{2}}{2}+2 \arctan \sqrt{2}=\sqrt{2} \sum_{k=1}^{\infty}(-1)^{k-1} 8^{-k}\left(\frac{12}{6 k-5}+\frac{2}{2 k-1}+\frac{3}{6 k-1}\right)$.

f)

$$
\begin{gathered}
S_{6}\left(\frac{\sqrt{2}}{2}\right)=\frac{\sqrt{2}}{6}\left(\arctan 2+(\sqrt{3}+1)\left(\arctan (-1)+\arctan \left(\frac{3+\sqrt{3}}{\sqrt{3}-1}\right)\right)+\right. \\
\left.+(\sqrt{3}-1)\left(\arctan (1)+\arctan \left(\frac{3-\sqrt{3}}{\sqrt{3}+1}\right)\right)\right)= \\
=\frac{\sqrt{2}}{6}\left(-\frac{\pi}{2}+\arctan (2)+(\sqrt{3}+1) \arctan \left(\frac{3+\sqrt{3}}{\sqrt{3}-1}\right)+(\sqrt{3}-1) \arctan \left(\frac{3-\sqrt{3}}{\sqrt{3}+1}\right)\right),
\end{gathered}
$$

hence, by applying the formulae for a sum and difference of arctangents, the equality, given below, follows

$$
\begin{gathered}
\frac{1}{3}\left(\left(\sqrt{3}-\frac{1}{2}\right) \pi+\arctan \left(\frac{3}{2}\right)+\arctan (2)-\sqrt{3} \arctan (2 \sqrt{3})\right)= \\
=\sum_{k=0}^{\infty} \frac{(-1)^{k}}{2^{6 k}}\left(\frac{1}{12 k+1}+\frac{1}{2(12 k+3)}+\frac{1}{2^{2}(12 k+5)}+\right. \\
\left.\quad+\frac{1}{2^{3}(12 k+7)}+\frac{1}{2^{4}(12 k+9)}+\frac{1}{2^{5}(12 k+11)}\right) .
\end{gathered}
$$


g)

$$
\begin{gathered}
S_{4}\left(\sin \frac{\pi}{8}\right)=\frac{\sqrt{2}}{2}\left(\sin \frac{\pi}{8} \arctan (2(\sqrt{2}-1))+\right. \\
\left.+\cos \frac{\pi}{8}(\arctan (2+\sqrt{2})-\arctan (\sqrt{2}))\right)= \\
=\frac{\sqrt{2}}{2}\left(\sin \frac{\pi}{8} \arctan (2(\sqrt{2}-1))+\cos \frac{\pi}{8} \arctan \left(\frac{2}{3+2 \sqrt{2}}\right)\right)
\end{gathered}
$$

which implies the equality

$$
\begin{gathered}
\frac{\sqrt{2}}{2}(\arctan (2(\sqrt{2}-1))+(\sqrt{2}+1) \arctan (2(3-2 \sqrt{2})))= \\
=\sum_{k=0}^{\infty}(-1)^{k} \frac{(2-\sqrt{2})^{4 k}}{2^{8 k}}\left(\frac{1}{8 k+1}+\frac{(2-\sqrt{2})}{2^{2}(8 k+3)}+\right. \\
\left.+\frac{(2-\sqrt{2})^{2}}{2^{4}(8 k+5)}+\frac{(2-\sqrt{2})^{3}}{2^{6}(8 k+7)}\right) .
\end{gathered}
$$

Similarly we get

$$
\begin{aligned}
S_{4}\left(\cos \frac{\pi}{8}\right) & =\frac{\sqrt{2}}{2}\left(\cos \frac{\pi}{8} \arctan (2(\sqrt{2}+1))+\sin \frac{\pi}{8}(\arctan (2-\sqrt{2})+\arctan (\sqrt{2}))\right)= \\
& =\frac{\sqrt{2}}{2}\left(\cos \frac{\pi}{8} \arctan (2(\sqrt{2}+1))+\sin \frac{\pi}{8} \arctan (2(3+2 \sqrt{2}))\right),
\end{aligned}
$$

from where we generate the formula in a way "conjugated" with the previous one

$$
\begin{gathered}
\frac{\sqrt{2}}{2}(\arctan (2(\sqrt{2}+1))+(\sqrt{2}-1) \arctan (2(3+2 \sqrt{2})))= \\
=\sum_{k=0}^{\infty}(-1)^{k} \frac{(2+\sqrt{2})^{4 k}}{2^{8 k}}\left(\frac{1}{8 k+1}+\frac{(2+\sqrt{2})}{2^{2}(8 k+3)}+\right. \\
\left.+\frac{(2+\sqrt{2})^{2}}{2^{4}(8 k+5)}+\frac{(2+\sqrt{2})^{3}}{2^{6}(8 k+7)}\right) .
\end{gathered}
$$

In both the last cases we used formulae [5, problem 1.19]:

$$
\begin{gathered}
\sin \frac{\pi}{8}=\frac{1}{2} \sqrt{2-\sqrt{2}}, \quad \cos \frac{\pi}{8}=\frac{1}{2} \sqrt{2+\sqrt{2}}, \quad \cos \frac{\pi}{8} \pm \sin \frac{\pi}{8}=\sqrt{\frac{2 \pm \sqrt{2}}{2}} \\
\tan \frac{\pi}{8}=\sqrt{2}-1, \quad 1-\cot \frac{\pi}{8}=-\sqrt{2}, \quad 1+\cot \frac{\pi}{8}=2+\sqrt{2} \\
\arctan x-\arctan y=\arctan \frac{x-y}{1+x y}, \text { whenever } x y>-1 .
\end{gathered}
$$


Remark 2. In Mathematica software the sums of series (16), (17) are presented with the use of hypergeometric functions which is much less attractive from the visually-technical point of view.

h)

$$
\begin{aligned}
S_{5}\left(\cos \frac{\pi}{5}\right)= & \frac{1}{5}\left((\sqrt{5}+1) \arctan \left(1+\frac{1}{\sqrt{5}}\right)+(\sqrt{5}-1) \arctan \left(2+\frac{4}{\sqrt{5}}\right)+\arctan \left(\frac{1+\sqrt{5}}{4}\right)\right)= \\
& =\sum_{n=0}^{\infty}(-1)^{n}\left(\frac{1+\sqrt{5}}{4}\right)^{10 n+1}\left(\frac{1}{10 n+1}+\frac{1}{10 n+3}\left(\frac{1+\sqrt{5}}{4}\right)^{2}+\right. \\
& \left.+\frac{1}{10 n+5}\left(\frac{1+\sqrt{5}}{4}\right)^{4}+\frac{1}{10 n+7}\left(\frac{1+\sqrt{5}}{4}\right)^{6}+\frac{1}{10 n+9}\left(\frac{1+\sqrt{5}}{4}\right)^{8}\right)
\end{aligned}
$$

in which we used relation [5, problem 1.18]:

$$
\begin{aligned}
\cos \frac{\pi}{5} & =\frac{1+\sqrt{5}}{4} \Rightarrow \sin \frac{\pi}{5}=\frac{\sqrt{10-2 \sqrt{5}}}{4} \\
\cos \frac{2 \pi}{5} & =\frac{\sqrt{5}-1}{4} \Rightarrow \sin \frac{2 \pi}{5}=\frac{\sqrt{10+2 \sqrt{5}}}{4} .
\end{aligned}
$$

\section{Generalizations of the Gregory-Leibniz-Nilakantha formula}

For series (3)-(10) obtained in Section 1 one can apply the Abel's Limit Theorem for power series $[6,7]$. As a result, we get the set of attractive formulae generalizing formula (2). For example, from $a$ ) we get

$$
\frac{1}{4} \sqrt{2} \pi=\sum_{k=0}^{\infty}(-1)^{k}\left(\frac{1}{4 k+1}+\frac{1}{4 k+3}\right) .
$$

We also receive

$$
3 S_{3}(1)=\lim _{x \rightarrow 1^{-}}\left(\arctan x-2 \arctan \frac{x}{x^{2}-1}\right)=\frac{\pi}{2}+\pi=\frac{3}{2} \pi,
$$

i.e.,

$$
\frac{\pi}{2}=\sum_{k=0}^{\infty}(-1)^{k}\left(\frac{1}{6 k+1}+\frac{1}{6 k+3}+\frac{1}{6 k+5}\right) .
$$

The next formulae are based on the following auxiliary relations

$$
\begin{gathered}
\lim _{x \rightarrow 1^{-}}(\arctan (x \csc \alpha-\cot \alpha)+\arctan (x \csc \alpha+\cot \alpha))= \\
=\lim _{x \rightarrow 1^{-}} \arctan \frac{2 x \csc \alpha}{1-x^{2} \csc ^{2}+\cot \alpha}=\lim _{x \rightarrow 1^{-}} \arctan \frac{2 x \sin \alpha}{1-x^{2}}=\frac{\pi}{2}, \quad \alpha \in(0, \pi)
\end{gathered}
$$


and

$$
\begin{gathered}
\lim _{x \rightarrow 1^{-}}(\arctan (x \sec \alpha-\tan \alpha)+\arctan (x \sec \alpha+\tan \alpha))= \\
=\lim _{x \rightarrow 1^{-}}\left(\arctan \left(x \csc \left(\frac{\pi}{2}-\alpha\right)-\cot \left(\frac{\pi}{2}-\alpha\right)\right)+\arctan \left(x \csc \left(\frac{\pi}{2}-\alpha\right)+\cot \left(\frac{\pi}{2}-\alpha\right)\right)\right)= \\
=\lim _{x \rightarrow 1^{-}} \arctan \frac{2 x \cos \alpha}{1-x^{2}}=\frac{\pi}{2}, \quad \alpha \in\left(-\frac{\pi}{2}, \frac{\pi}{2}\right) .
\end{gathered}
$$

Hence, from (6) we deduce

$$
\begin{aligned}
S_{4}(1)= & \frac{\sqrt{2}}{2}\left(\cos \frac{\pi}{8} \cdot \frac{\pi}{2}+\sin \frac{\pi}{8} \cdot \frac{\pi}{2}\right)=\frac{\sqrt{2} \pi}{4}\left(\cos \frac{\pi}{8}+\sin \frac{\pi}{8}\right)= \\
& \stackrel{(19))}{=} \frac{\sqrt{2} \pi}{8}(\sqrt{2-\sqrt{2}}+\sqrt{2+\sqrt{2}})=\frac{\pi}{4} \sqrt{2+\sqrt{2}}
\end{aligned}
$$

which implies

$$
\frac{\pi}{4} \sqrt{2+\sqrt{2}}=\sum_{k=0}^{\infty}(-1)^{k}\left(\frac{1}{8 k+1}+\frac{1}{8 k+3}+\frac{1}{8 k+5}+\frac{1}{8 k+7}\right)
$$

Whereas, from (7) we obtain

$$
\begin{gathered}
5 S_{5}(1)=\frac{\pi}{4}+4 \cos \frac{2 \pi}{5} \cdot \frac{\pi}{2}+4 \cos \frac{\pi}{5} \cdot \frac{\pi}{2}=2 \pi\left(\frac{1}{8}+\cos \frac{\pi}{5}+\cos \frac{2 \pi}{5}\right)= \\
\stackrel{(20))}{=} 2 \pi\left(\frac{1}{8}+\frac{\sqrt{5}}{2}\right)=\pi\left(\frac{1}{4}+\sqrt{5}\right),
\end{gathered}
$$

which implies

$$
\begin{gathered}
\frac{\pi}{5}\left(\frac{1}{4}+\sqrt{5}\right)=\sum_{k=0}^{\infty}(-1)^{k}\left(\sum_{r=1}^{5} \frac{1}{10 k+2 r-1}\right)= \\
=\sum_{k=0}^{\infty}(-1)^{k}\left(H_{10 k+9}^{*}-H_{10 k-1}^{*}\right)
\end{gathered}
$$

where $H_{2 k-1}^{*}$ denotes the $k$-th odd harmonic number announced in the Introduction

$$
H_{2 k-1}^{*}:=\sum_{l=1}^{k} \frac{1}{2 l-1}, \quad k=1,2, \ldots
$$

From (8) we get

$$
3 \sqrt{2} S_{6}(1)=\frac{\sqrt{2}}{4} \pi+\sqrt{3} \pi=\left(\frac{\sqrt{2}}{4}+\sqrt{3}\right) \pi
$$


i.e.,

$$
\frac{\pi}{6}\left(\frac{1}{2}+\sqrt{6}\right)=\sum_{k=0}^{\infty}(-1)^{k}\left(H_{12 k+11}^{*}-H_{12 k-1}^{*}\right) .
$$

From (9) we obtain

$$
7 S_{7}(1)=\frac{\pi}{4}+\frac{\pi}{2}\left(1+6 \cos \frac{\pi}{7}-2 \sin \frac{\pi}{14}+2 \sin \frac{3 \pi}{14}\right)
$$

which gives us the "beautiful" formula

$$
\frac{\pi}{7}\left(\frac{3}{4}+3 \cos \frac{\pi}{7}-\sin \frac{\pi}{14}+\sin \frac{3 \pi}{14}\right)=\sum_{k=0}^{\infty}(-1)^{k}\left(H_{14 k+13}^{*}-H_{14 k-1}^{*}\right) .
$$

At last, from (10) we receive

$$
\frac{9}{2} S_{9}(1)=\frac{\pi}{8}+\frac{\pi}{2}\left(2+\cos \frac{\pi}{9}+3 \cos \frac{2 \pi}{9}-\sin \frac{\pi}{18}\right) .
$$

Hence, the next our "lovely" formula follows

$$
\frac{\pi}{9}\left(\frac{9}{4}+\cos \frac{\pi}{9}+3 \cos \frac{2 \pi}{9}-\sin \frac{\pi}{18}\right)=\sum_{k=0}^{\infty}(-1)^{k}\left(H_{18 k+17}^{*}-H_{18 k-1}^{*}\right) .
$$

Remark 3. Numbers $\cos \frac{\pi}{7}, \sin \frac{\pi}{14}=\cos \frac{3 \pi}{7}, \sin \frac{3 \pi}{14}=\cos \frac{2 \pi}{7}$ from (25) are linearly independent over $\mathbb{Q}$. Similarly, numbers $\cos \frac{\pi}{9}, \cos \frac{2 \pi}{9}$ and $\sin \frac{\pi}{18}=\cos \frac{4 \pi}{9}$ are also linearly independent over $\mathbb{Q}$. In both cases, with regard to the appropriate trigonometric identities, it means that the rational linear combinations of these numbers cannot be rational numbers either. In this connection, it seems to be unlikely to reduce the number of sums of components on the left sides of formulae (25) and (26).

Final comments. Additional historical remarks concerning Gregory can be found in [8]. Formulae related to the sums of series, presented in this paper, were verified with [6], [9], [10] and [11]. The greater part of these formulae seems to be original, especially original is the method of generating the discussed formulae.

\section{References}

[1] Boyer C.B., revised by Merzbach U.C., A History of Mathematics, Wiley, New York 1991.

[2] Wituła R., Number $\pi$, its history and influence on mathematical creativity, Wyd. Prac. Komputerowej J. Skalmierskiego, Gliwice 2011 (in Polish). 
[3] Wituła R., Słota D., On the sum of some alternating series, Comput. Math. Appl. 2011, 62, 2658-2664.

[4] Modenov P.S., Collection of Problems for a Special Course of Elementary Mathematics, Sowietskaja Nauka, Moskva 1957 (in Russian).

[5] Wituła R., Complex Numbers, Polynomials and Partial Fractions Decompositions, Part I, Wyd. Pol. Śl., Gliwice 2010 (in Polish).

[6] Knopp K., Infinite Series, PWN, Warsaw 1956 (in Polish).

[7] Wituła R., On certain applications of formulae for sum of unimodular complex numbers, Wyd. Prac. Komputerowej J. Skalmierskiego, Gliwice 2011 (in Polish).

[8] Edwards C.H., The Historical Development of the Calculus, Springer, New York 1979.

[9] Prudnikov A.P., Brychkov Yu.A., Marichev O.I., Integrals and Series, Vol. 1, Elementary Functions, Gordon \& Breach Sci. Pub., New York 1986.

[10] Bromwich T.J.I'A., An Introduction to the Theory of Infinite Series, Merchant Books 2008.

[11] Prus-Wiśniowski F., Real Series, Wyd. Nauk. Uniw. Szczecińskiego, Szczecin 2005 (in Polish). 